\title{
Defect Engineering of Nanoscale Hf-based Metal-organic frameworks for Highly Efficient Iodine Capture
}

Yuyao Guan, ${ }^{a}$ Yite Li, ${ }^{b, c}$ Junli Zhou, ${ }^{b, c}$ Tao Zhang, ${ }^{d}$ Jun Ding, ${ }^{*}, a$ Zhigang Xie, ${ }^{b, c}$ Lei Wang ${ }^{*}, b$

${ }^{a}$ Department of Radiology, China-Japan Union Hospital of Jilin University, 126

Xiantai Street, Changchun, Jilin 130033, P. R. China.

${ }^{b}$ State Key Laboratory of Polymer Physics and Chemistry, Changchun Institute of Applied Chemistry, Chinese Academy of Sciences, 5625 Renmin Street, Changchun, Jilin 130022, P. R. China.

${ }^{c}$ University of Science and Technology of China, Hefei, Anhui 230026, P. R. China.

${ }^{d}$ Department of Gastrointestinal Colorectal and Anal Surgery, China-Japan Union Hospital of Jilin University, 126 Xiantai Street, Changchun, Jilin 130033, P. R. China

*Corresponding author.E-mail: leiwang@ciac.ac.cn E-mail:dingjun@jlu.edu.cn 


\section{Table of Contents}

Section 1 HUN and DHUN synthesis, element distribution and SEM, TEM, DLS detection

Section 2 Color change of iodine/cyclohexane solution of HUN and DHUN adsorbing iodine

Section 3 Element distribution, SEM, TEM, DLS detection after HUN and DHUN adsorption and desorption of iodine

Section 4 Powder X-ray diffraction and thermogravimetric analysis 11

Section 5 Raman spectroscopy, XPS and Fourier transform infrared spectroscopy 13

Reference 16 
Section 1 HUN and DHUN synthesis, element distribution and HUN SEM, TEM, DLS detection

\subsection{Chemicals and materials}

Hafnium chloride $\left(\mathrm{HfCl}_{4}\right)$, Benzoic acid (BA), and cyclohexane were obtained from Beijing Chemical Co., Ltd. Terephthalic acid was purchased from Shanghai Sinopharm Chemical Reagent Co., Ltd. All reagents and solvents can be used without further purification.

\subsection{Synthesis of HUN and DHUN}

The preparation of Hf-UiO-66 refers to previous reports, ${ }^{1,2}$ and is named HUN in this article. $\mathrm{HfCl}_{4}(106 \mathrm{mg}), \mathrm{BDC}(52 \mathrm{mg}), \mathrm{BA}(230 \mathrm{mg})$, and $\mathrm{HCl}(37 \%, 55 \mathrm{uL})$ were mixed in DMF (6 mL), disperse the mixture uniformly under ultrasound, move it into the reactor, and heat it in the oven for 24 hours at $120^{\circ} \mathrm{C}$. After cooling, the white powder of HUN was collected by centrifugation and washed twice with DMF and methanol, respectively.

For defective Hf-MOF (DHUN): HUN (40 mg), HCl (37\%, $1 \mathrm{~mL})$, and DMF (20 $\mathrm{mL}$ ) were mixed in the sample bottle and evenly dispersed by ultrasonic. The sample was heated in the oven at $60{ }^{\circ} \mathrm{C}$ for 10 hours. After cooling, the white powder of defected Hf-MOF was collected by centrifugation and washed twice with DMF and methanol, respectively. 
The ${ }^{1} \mathrm{H}$ NMR spectrum of the target product recorded on a Bruker NMR-400 DRX spectrometer at room temperature. The ${ }^{1} \mathrm{H}$ NMR spectrum showed that the hydrogen peak of terephthalic acid at $7.9 \mathrm{ppm}$ was present, while the hydrogen peak of benzoic acid at $7.5 \mathrm{ppm}$ disappeared (Figure S1), indicating that we successfully created the defect site.

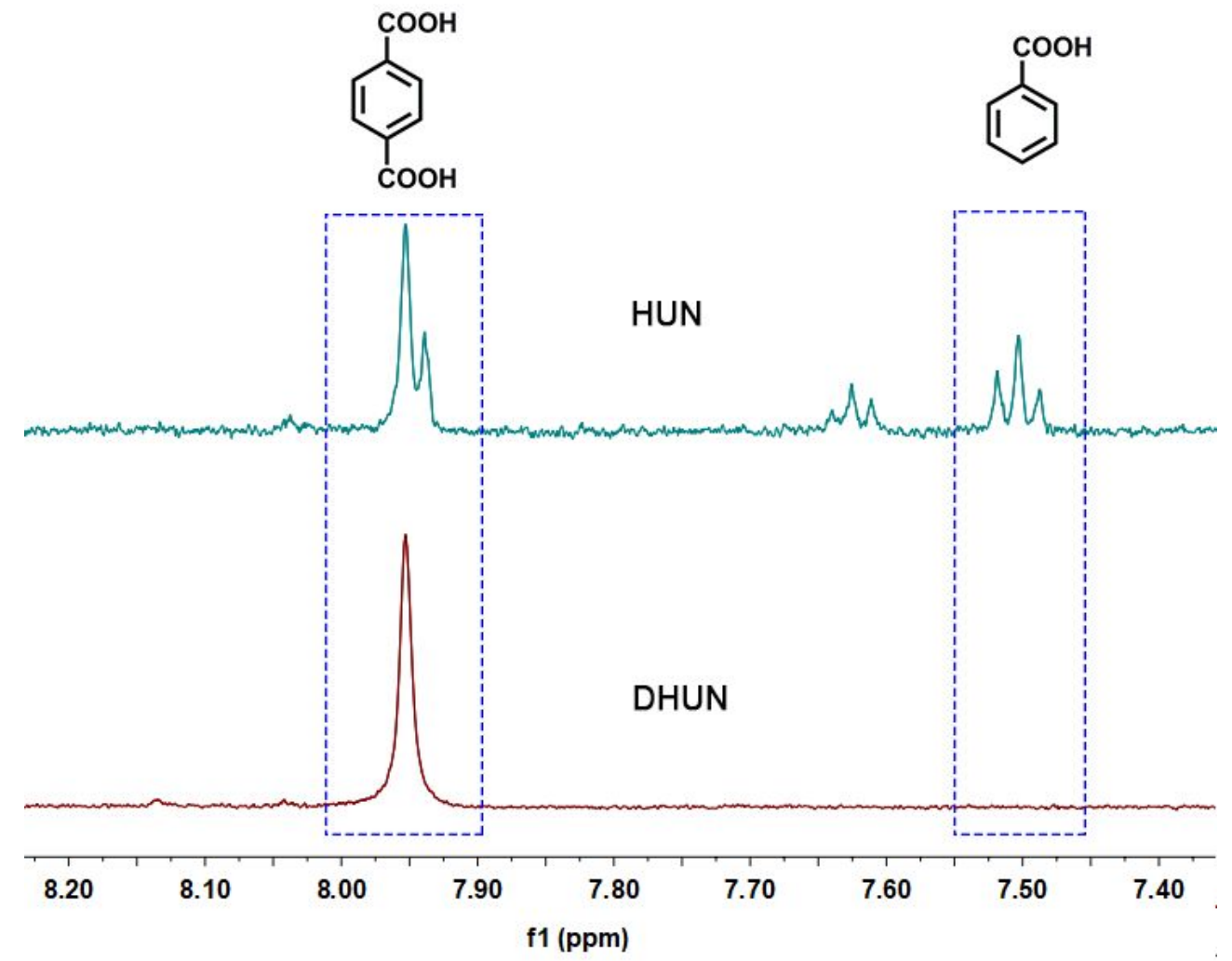

Figure S1. The ${ }^{1} \mathrm{H}$ NMR spectra of HUN and DHUN. 


\subsection{Basic characterizations}

The size of the prepared HUN was studied by a transmission electron microscope (TEM) under a JEOL JEM-1011 electron microscope with an accelerating voltage of $100 \mathrm{kV}$. The morphology of HUN was also studied using a field emission scanning electron microscope (FE-SEM, S-4800, Hitachi) as shown in Figure S2b. HUN is a regular octahedron. The DLS of HUN is inserted in Figure S2a. The element distribution of HUN and DHUN is shown in Figures S2c and S2d, and there is no obvious difference in the distribution of $\mathrm{C}, \mathrm{O}$, and $\mathrm{Hf}$.

a)

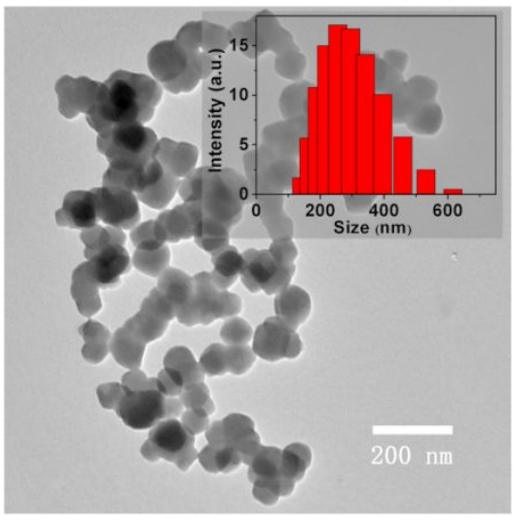

c)

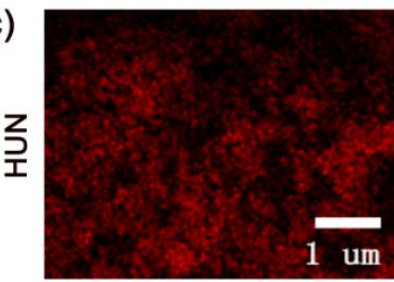

d)

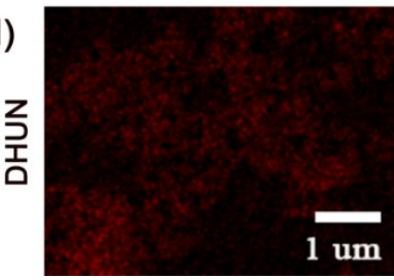

b)
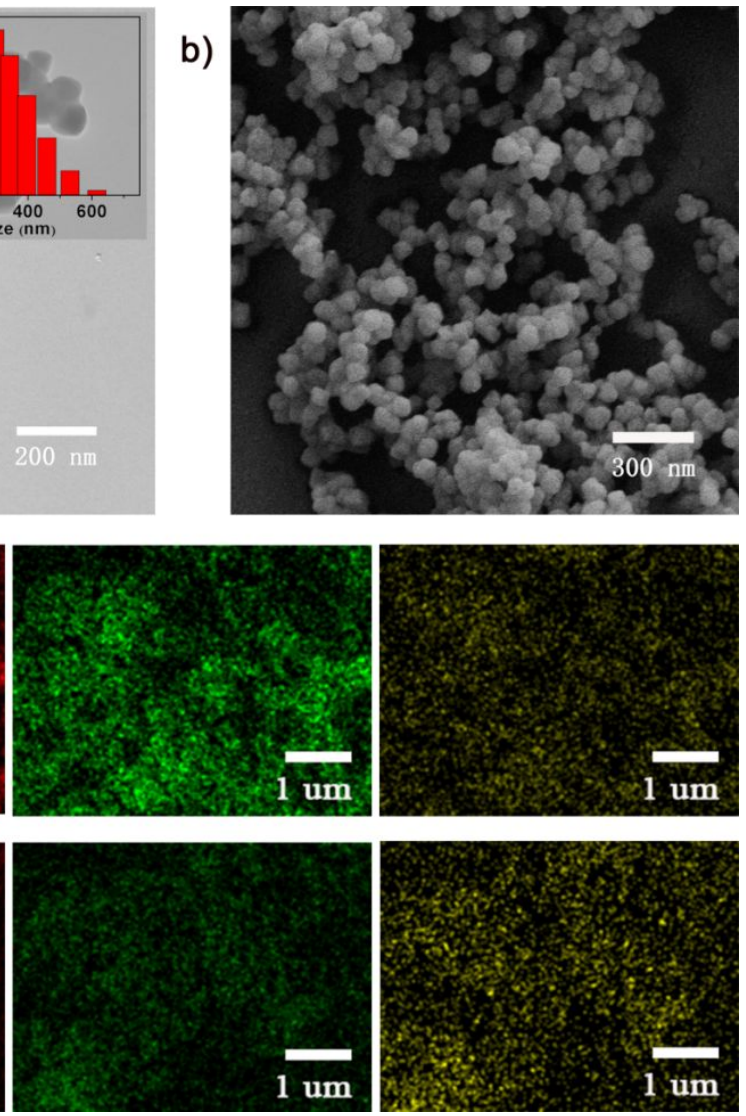

Figure S2. (a) TEM and corresponding DLS image of HUN. Scale bar: $200 \mathrm{~nm}$. (b)

SEM image of HUN (scale bar, $300 \mathrm{~nm}$ ). (c) Element mapping (scale bar, $1 \mathrm{um}$ ) of HUN. (d) Element mapping (scale bar, 1 um) of DHUN. 


\section{Section 2 Color change of iodine/cyclohexane solution of HUN and DHUN after adsorption and desorption of iodine}

Iodine adsorption: $10 \mathrm{mg}$ of HUN or DHUN sample were placed into each sample bottle, and then added $2 \mathrm{~mL}$ of iodine cyclohexane solution $(0.001 \mathrm{~mol} / \mathrm{L})$ to seal it at room temperature for $72 \mathrm{~h}$. The color of the iodine/cyclohexane solution of HUN gradually changed from dark purple to almost colorless, while the color of the iodine/cyclohexane solution of DHUN changed from dark purple to more colorless (Figure S3). The adsorption kinetics was studied by monitoring the UV absorbance of the solution at $523 \mathrm{~nm}$ at different time points.

Iodine release: Add samples $(10 \mathrm{mg})$ of saturated iodine to ethanol solution, and place them at room temperature for $50 \mathrm{~min}$ for iodine release. The release kinetics was studied by monitoring the absorbance at $357 \mathrm{~nm}$ at different time points by UV-vis spectroscopy. 


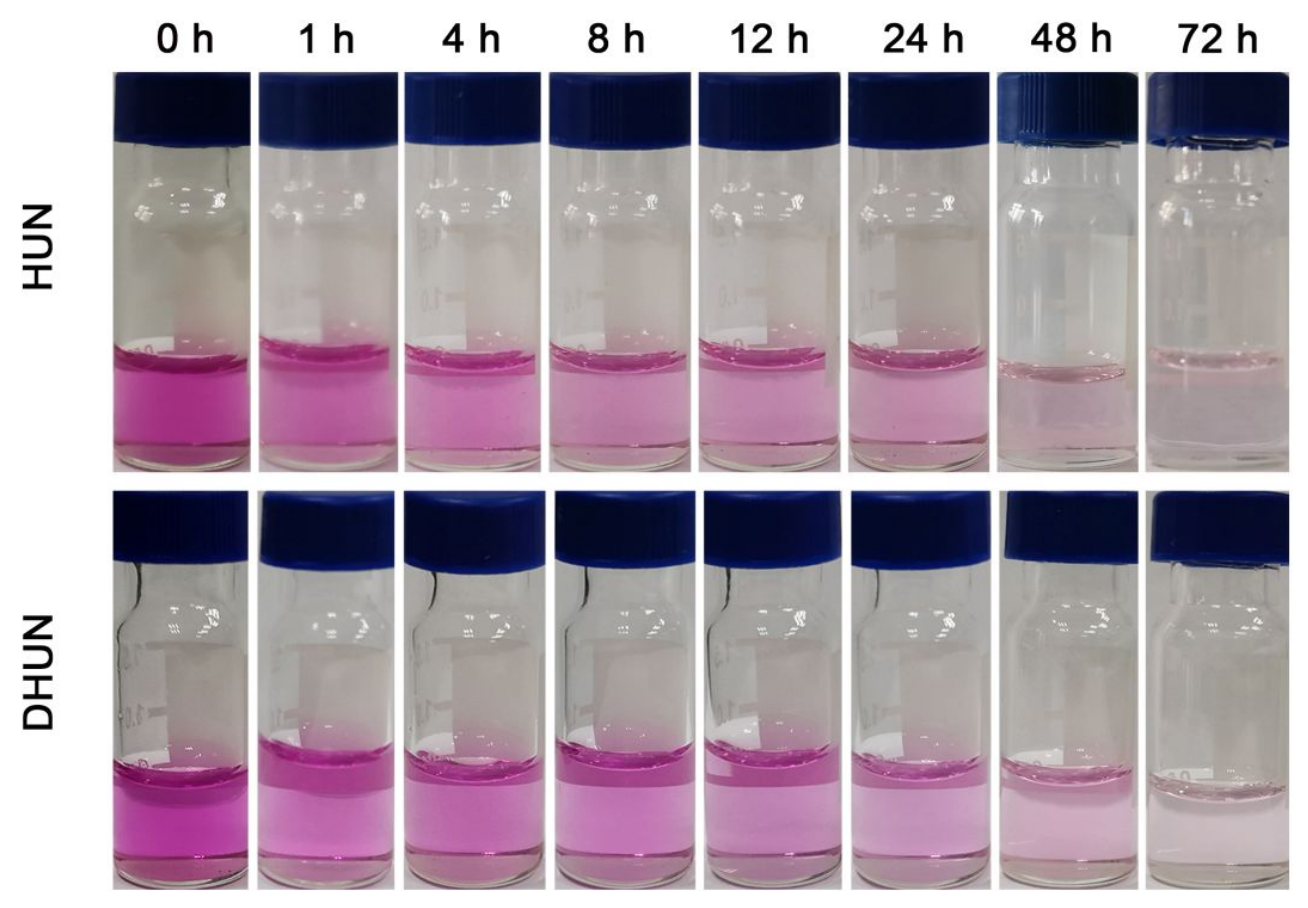

Figure S3. The color changes of the iodine/cyclohexane solution changes during the adsorption of iodine by HUN and DHUN from 0 to $72 \mathrm{~h}$. 
Section 3 Element distribution, SEM, TEM, DLS detection of HUN and DHUN after adsorption and desorption of iodine

a)

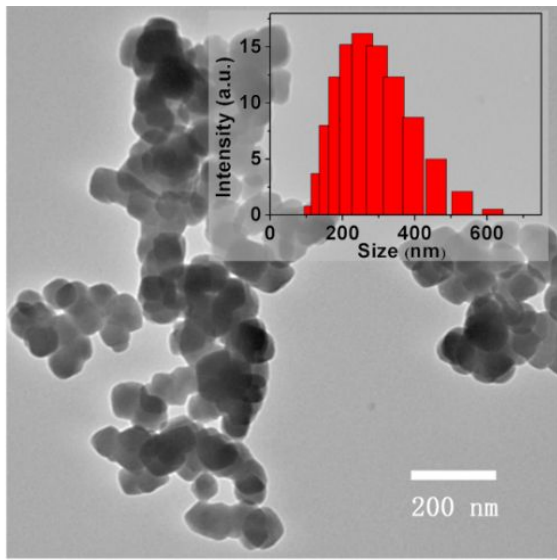

c)
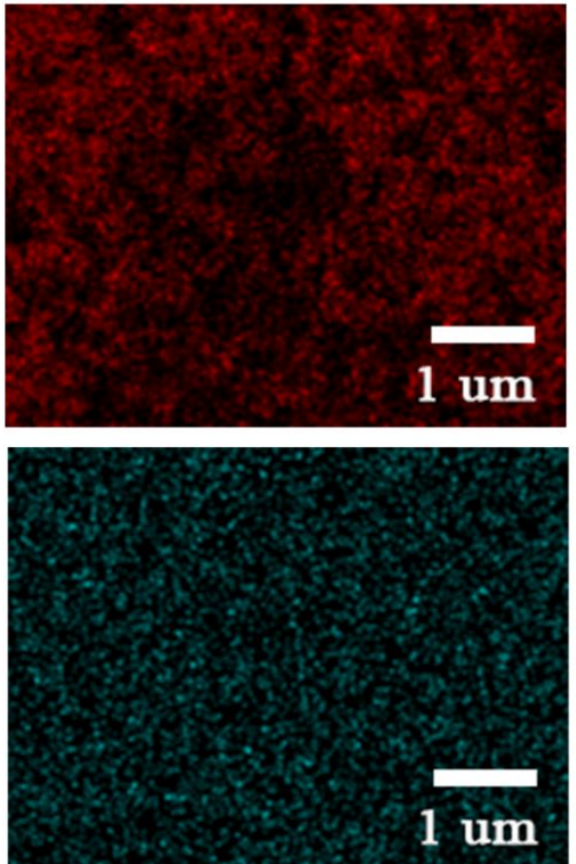

b)
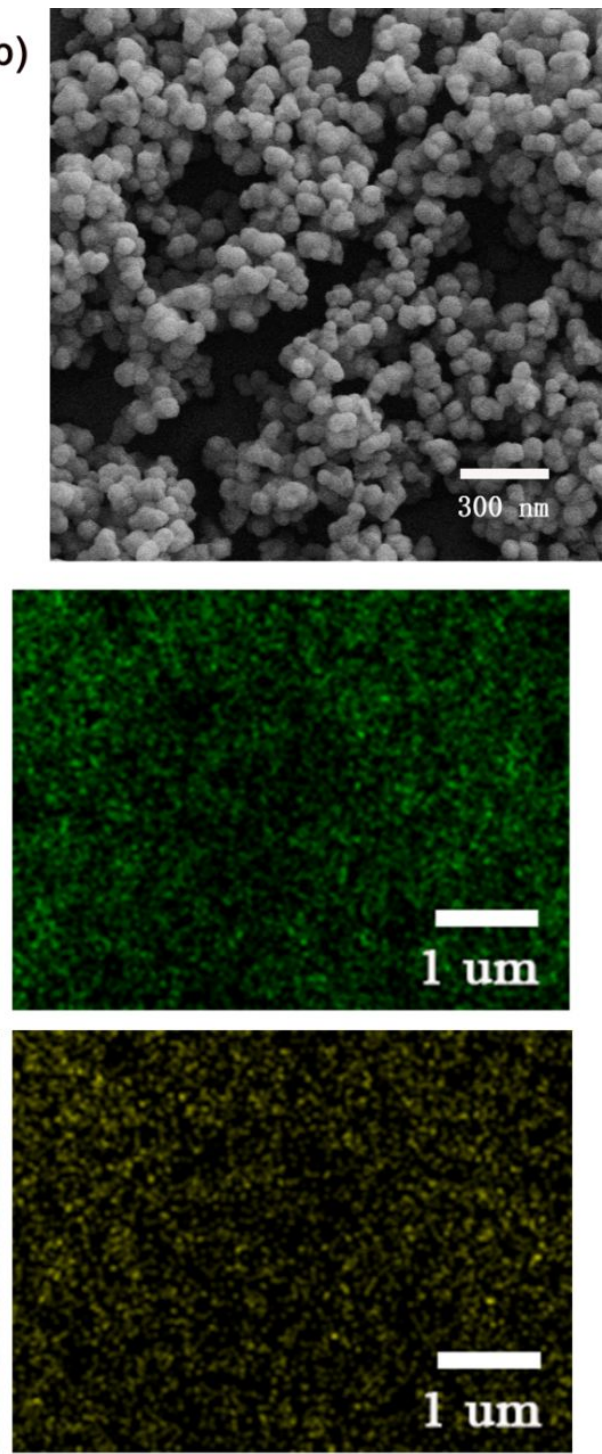

Figure S4. (a) TEM image (scale bar, $200 \mathrm{~nm}$ ) and corresponding DLS of $\mathrm{I}_{2 \mathrm{a}} @ H U N$.

(b) SEM image of $\mathrm{I}_{2 \mathrm{a}} @ \mathrm{HUN}$ (scale bar, 300 nm). (c) Element mapping (scale bar, 1 um) of $\mathrm{I}_{2 \mathrm{a}} @ \mathrm{HUN}$. 
a)

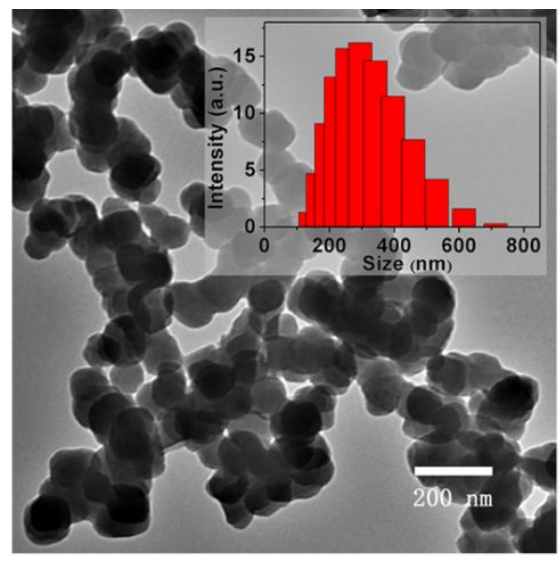

c)
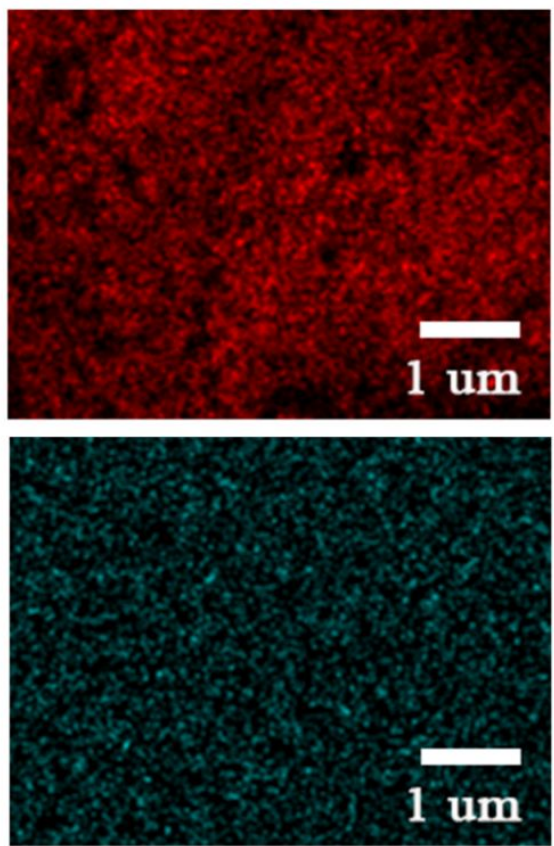

b)
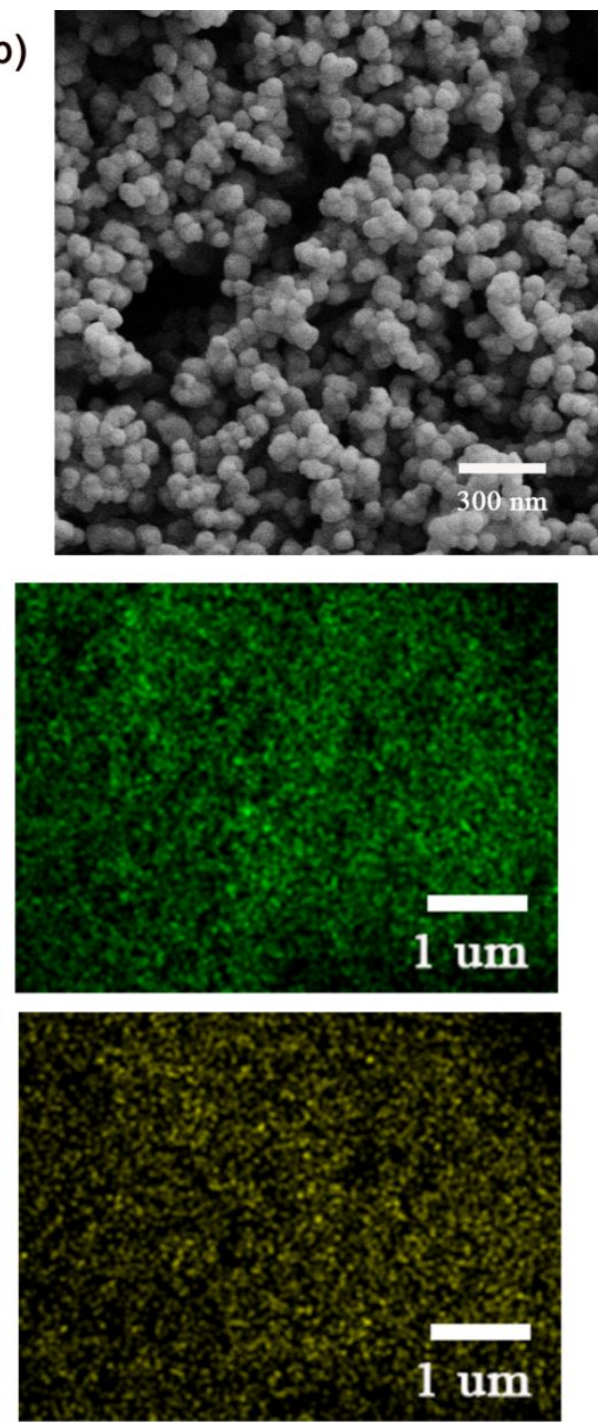

Figure S5. (a) TEM (scale bar, $200 \mathrm{~nm}$ ) and corresponding DLS of $\mathrm{I}_{2 \mathrm{c}} @ H U N$. (b)

SEM image of $\mathrm{I}_{2 \mathrm{c}} @ \mathrm{HUN}$ (scale bar, 300 nm). (c) Element mapping (scale bar, $1 \mathrm{um}$ ) of $\mathrm{I}_{2 \mathrm{c}} @ \mathrm{HUN}$. 
a)

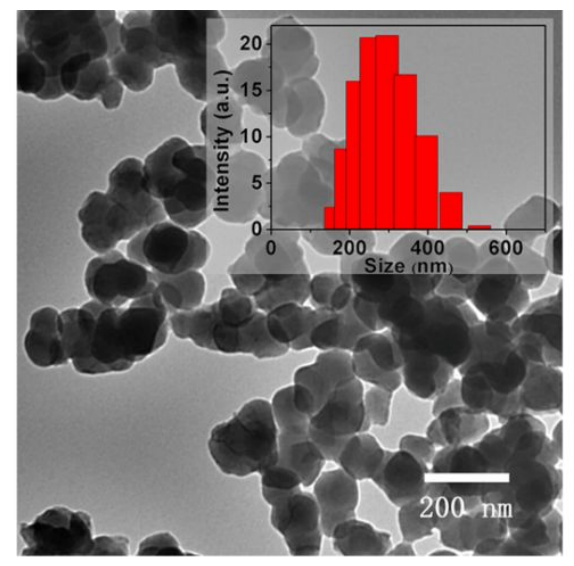

c)
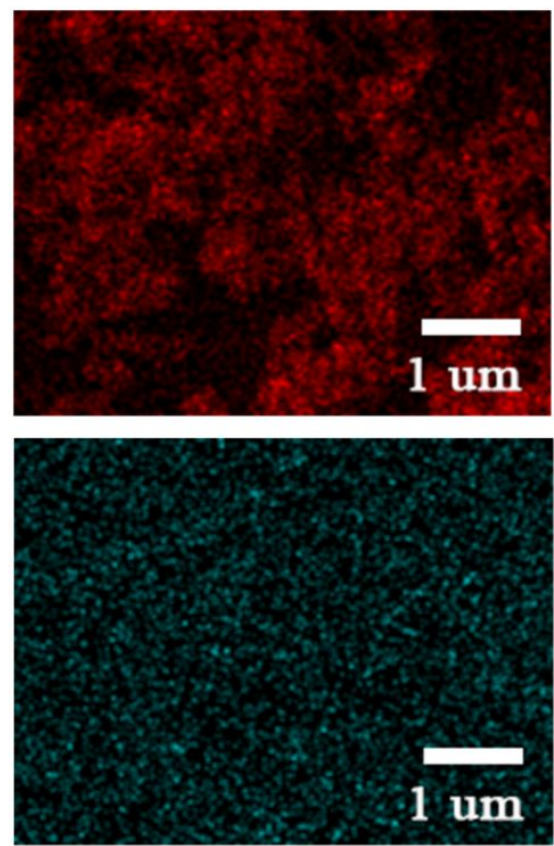

b)
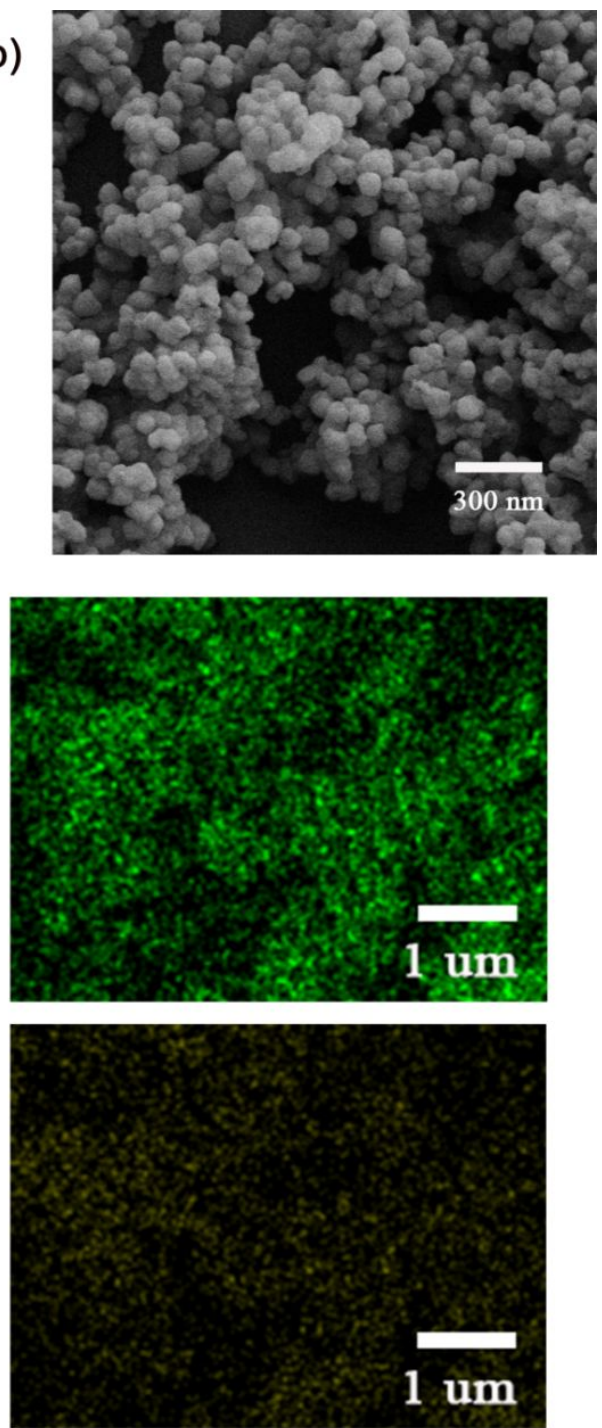

Figure S6. (a) TEM (scale bar, $200 \mathrm{~nm}$ ) and corresponding DLS of $\mathrm{I}_{2 \mathrm{a}} @$ DHUN. (b)

SEM image of $\mathrm{I}_{2 \mathrm{a}} @$ DHUN (scale bar, $300 \mathrm{~nm}$ ). (c) Element mapping (scale bar, 1 um) of $I_{2 a} @ D H U N$. 


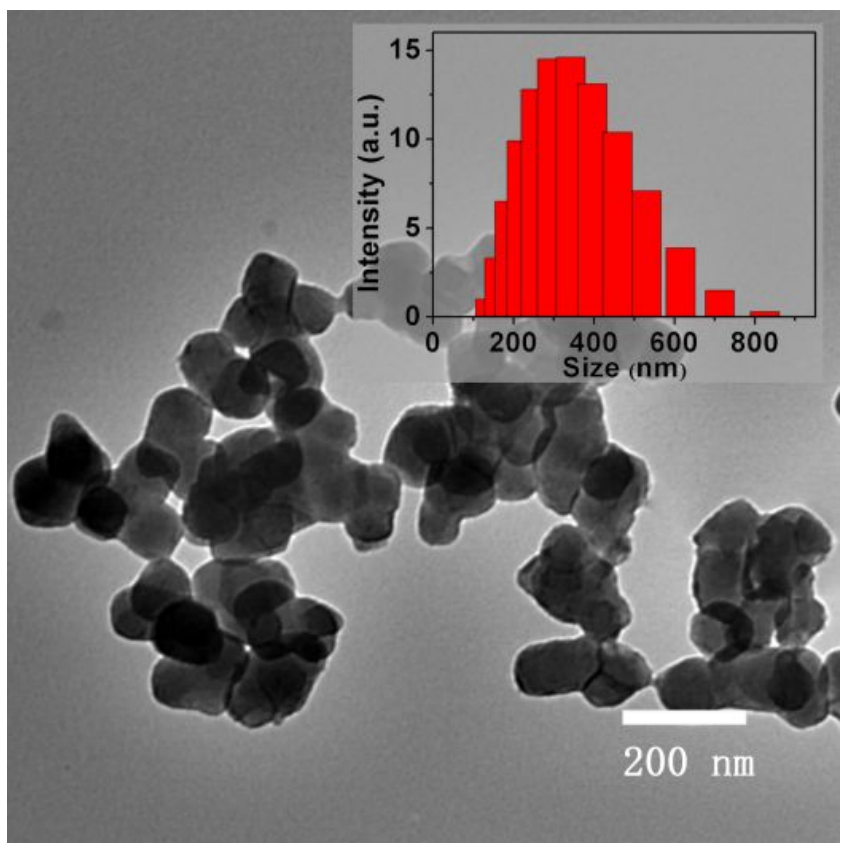

Figure S7. TEM and corresponding DLS of $\mathrm{I}_{2 \mathrm{c}} @ \mathrm{DHUN}$, scale bar, $200 \mathrm{~nm}$. 


\section{Section 4 Powder X-ray diffraction and thermogravimetric analysis}

Powder X-ray diffraction (PXRD) was obtained by Bruker D8 Advance measuring instrument to study the crystal structure of HUN, $\mathrm{I}_{2 \mathrm{a}} @ \mathrm{HUN}, \mathrm{I}_{2 \mathrm{c}} @ \mathrm{HUN}$ (Figure S8). We found that the PXRD peak intensity and position after HUN adsorption and desorption of iodine did not change significantly, which also shows that the capture of iodine did not destroy the integrity of the crystal structure.

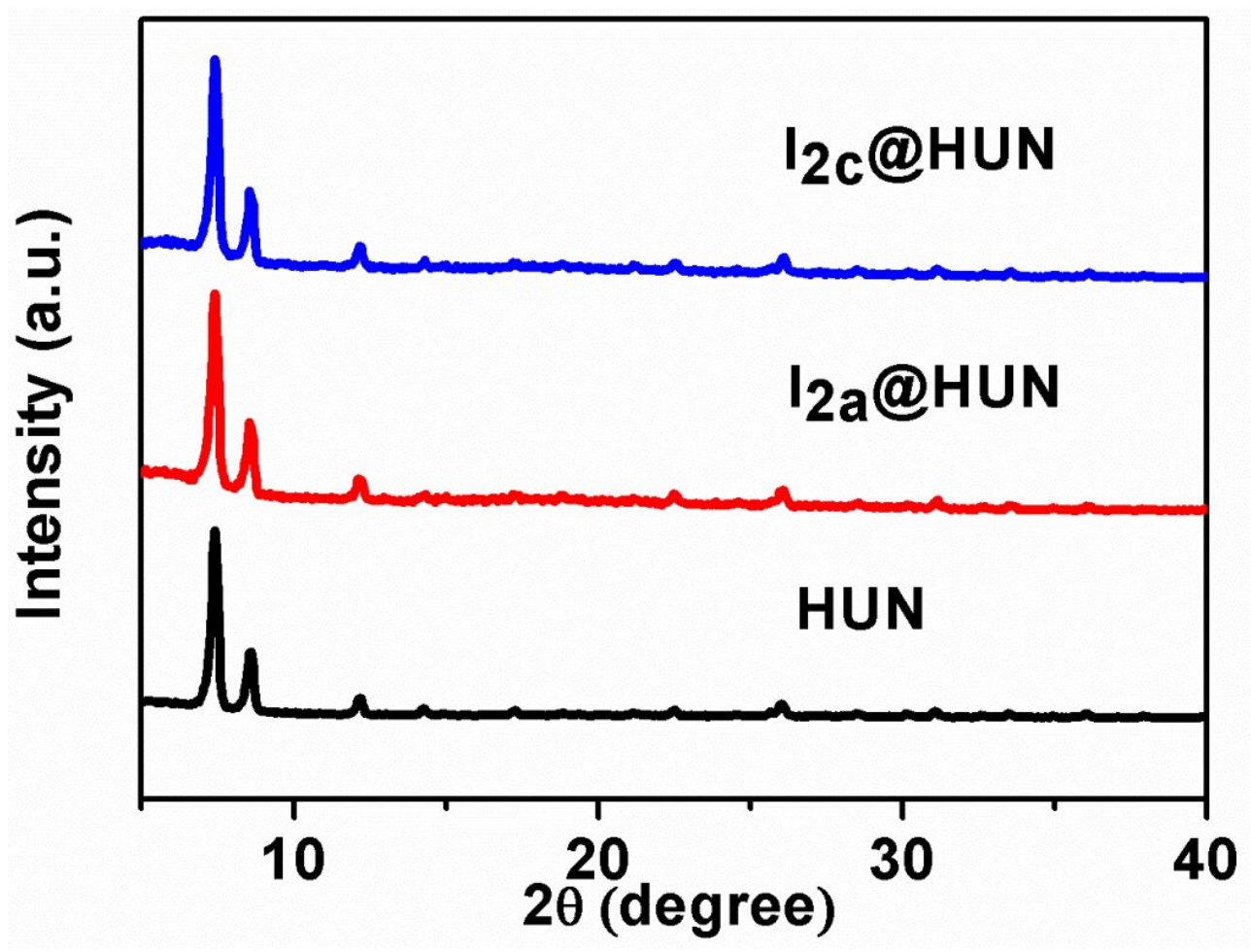

Figure S8. PXRD patterns of HUN, $\mathrm{I}_{2 \mathrm{a}} @ \mathrm{HUN}$ and $\mathrm{I}_{2 \mathrm{c}} @ \mathrm{HUN}$ powder. 
The thermogravimetric measurements (Pyris Diamond TGA/DTA) of all samples were measured at room temperature to $800^{\circ} \mathrm{C}$ and $10^{\circ} \mathrm{C} / \mathrm{min}$ under air conditions (Figure S9). At $200{ }^{\circ} \mathrm{C}$, the weight loss rate of $\mathrm{I}_{2 \mathrm{c}} @ \mathrm{HUN}$ was 9.8\%. When the temperature reached $500{ }^{\circ} \mathrm{C}$, the weight loss was $17.9 \%$. Finally, the weight loss rate reached $49.1 \%$ at $800{ }^{\circ} \mathrm{C}$. As for $\mathrm{I}_{2 \mathrm{c}} @ \mathrm{DHUN}$, the weight loss rate was $18.7 \%$ at $200{ }^{\circ} \mathrm{C}$, and then the mass loss was $32.3 \%$ at $500{ }^{\circ} \mathrm{C}$. While the weight loss rate of $\mathrm{I}_{2 \mathrm{c}} @$ DHUN was $50.3 \%$ at $800{ }^{\circ} \mathrm{C}$.
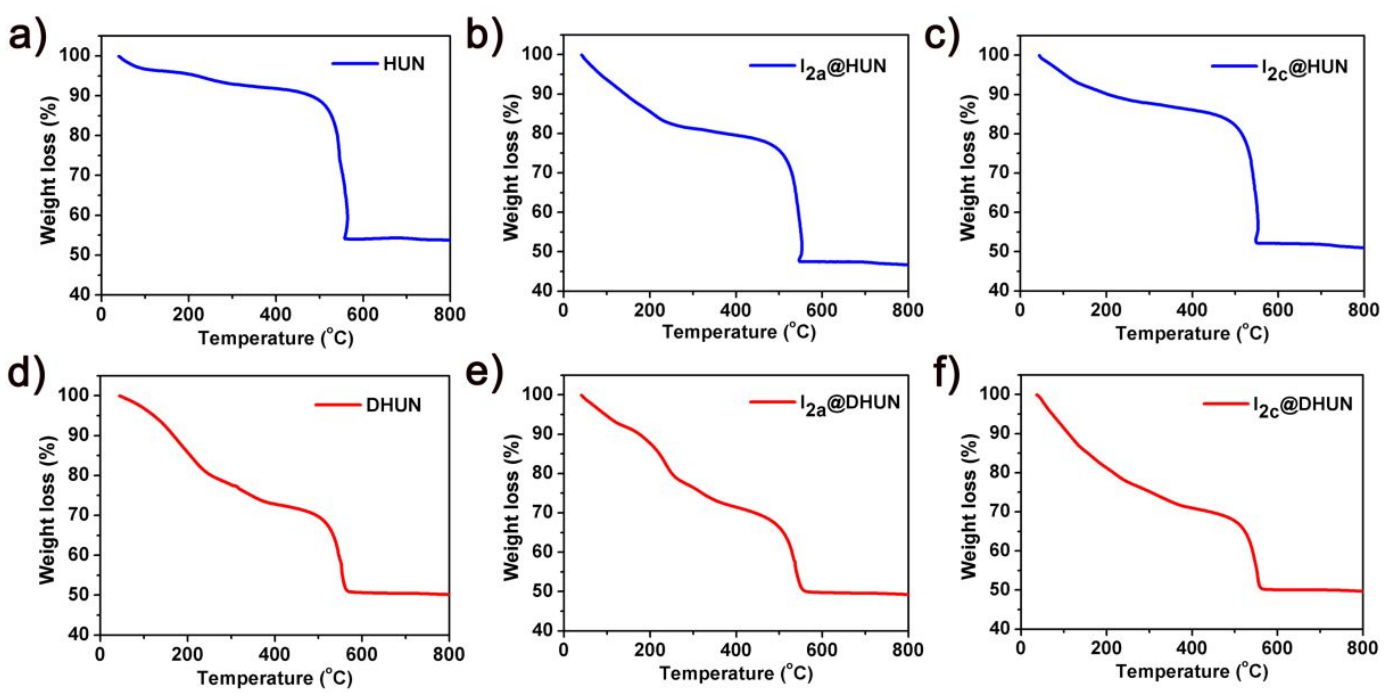

Figure S9. Thermogravimetric analysis (TGA) of (a) HUN, (b) $\mathrm{I}_{2 \mathrm{a}} @ \mathrm{HUN}$, (c) I $2 @$ HUN, (d) DHUN, (e) I $\mathrm{2a}_{\mathrm{a}} @ D H U N,(\mathrm{f}) \mathrm{I}_{2 \mathrm{c}} @ \mathrm{DHUN}$ in air.

Table S1. Iodine loadings of HUN and DHUN.

\begin{tabular}{ccccc}
\hline & $\mathbf{I}_{\mathbf{2} \mathbf{a}} @ \mathbf{H U N}$ & $\mathbf{I}_{\mathbf{2} \mathbf{c}} @ \mathbf{H U N}$ & $\mathbf{I}_{\mathbf{2} \mathbf{a}} @ \mathbf{D H U N}$ & $\mathbf{I}_{\mathbf{2} \mathbf{c}} @ \mathbf{D H U N}$ \\
\hline $\mathbf{m g} / \mathbf{g}$ & 90 & 60 & 130 & 96 \\
\hline
\end{tabular}


Section 5 Raman spectroscopy, XPS and Fourier transform infrared spectroscopy

Raman microscope (HORIBA, LabRAM HR Evolution, excitation wavelength 532 $\mathrm{nm}$ ) was used to analyze the properties of iodine trapped on NMOF samples. As shown in Figure S10, HUN has no obvious peak between $100-200 \mathrm{~cm}^{-1}$, while $\mathrm{I}_{2 \mathrm{a}} @ \mathrm{HUN}$ and $\mathrm{I}_{2 \mathrm{c}} @ \mathrm{HUN}$ can see obvious peaks at $110 \mathrm{~cm}^{-1}$. It is considered that during the iodine adsorption process, a part of $\mathrm{I}_{2}$ converted to $\mathrm{I}_{3}{ }^{-}$.

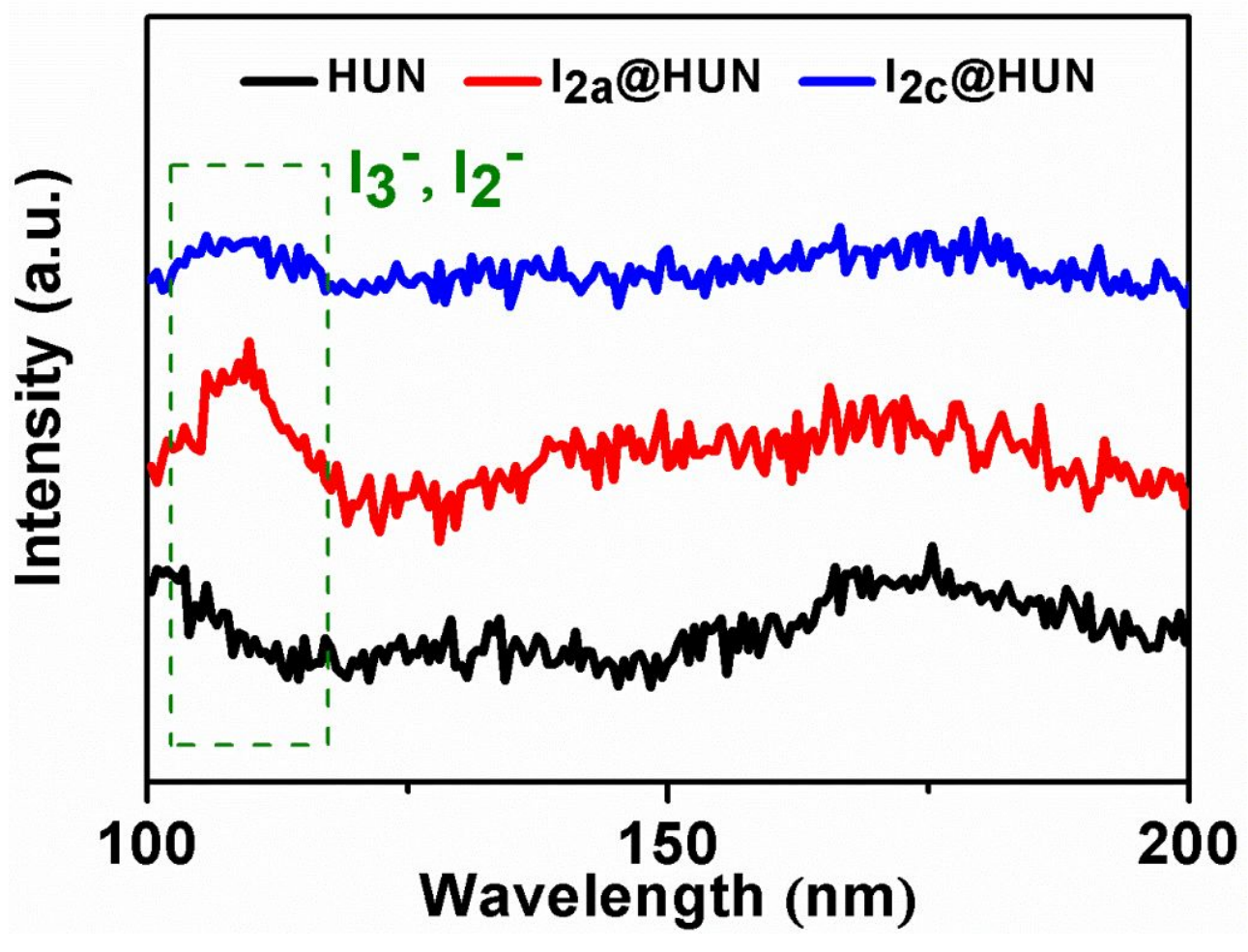

Figure S10. Raman spectra of HUN, $\mathrm{I}_{2 a} @ H U N$ and $\mathrm{I}_{2 c} @ H U N$. 
XPS (Thermo Scientific ESCALAB 250) was also used to analyze the properties of iodine trapped on NMOF samples. Figure S11 and S12 show the XPS spectra of DHUN and $I_{2 a} @ D H U N$, respectively. The XPS spectra of $\mathrm{C} 1 \mathrm{~s}$ and $\mathrm{O} 1 \mathrm{~s}$ of $\mathrm{I}_{2 \mathrm{a}} @$ DHUN did not change significantly compared with DHUN.

a)

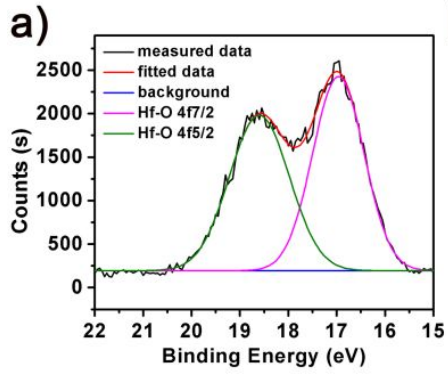

b)

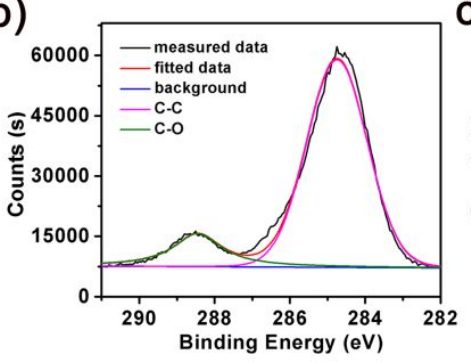

c)

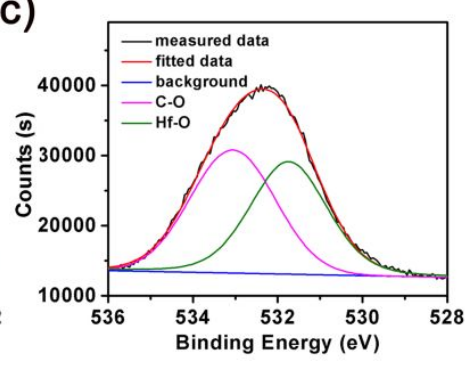

Figure S11. High-resolution XPS of DHUN (a) Hf 4f, (b) C 1s, (c) O 1s.

a)

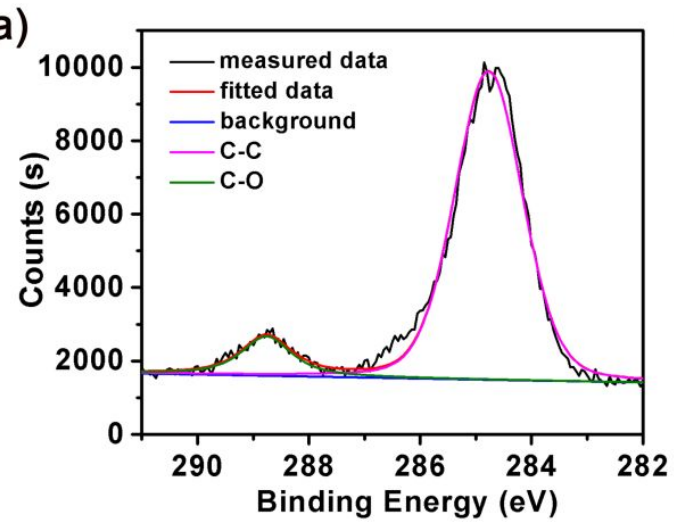

b)

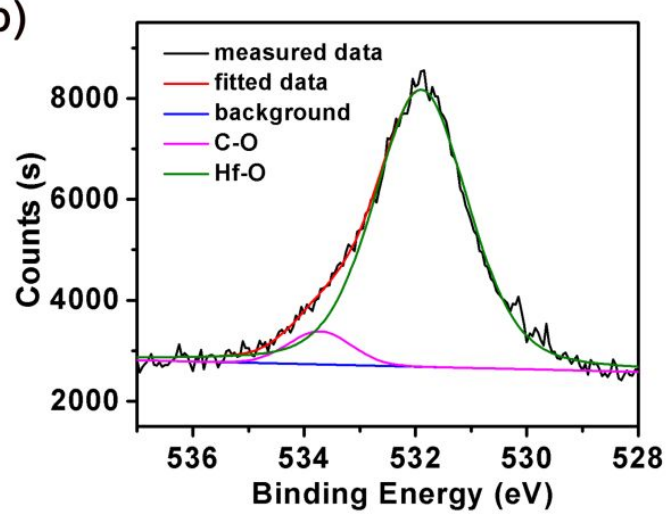

Figure S12. High-resolution XPS of $\mathrm{I}_{2 \mathrm{a}} @ D H U N$ (a) C 1s, (b) O 1s. 
The Fourier transform infrared (FT-IR) spectra of HUN and DHUN before and after the adsorption and desorption of iodine were recorded on a Bruker Vertex 70 spectrometer between 4000 and $500 \mathrm{~cm}^{-1}$.

a)

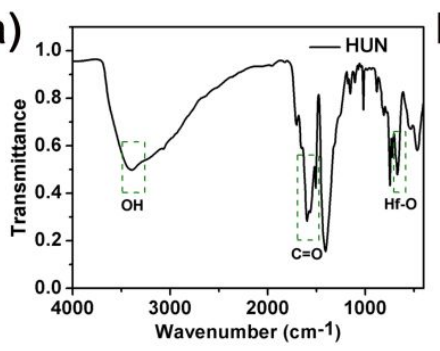

b)

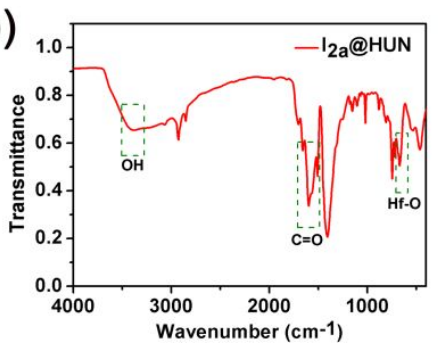

c)

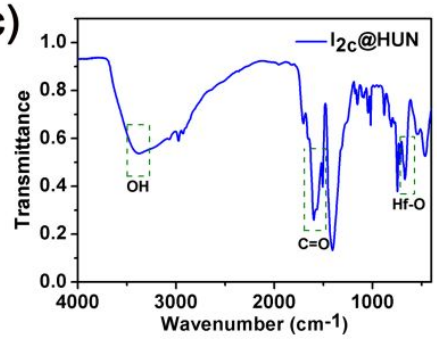

Figure S13. FTIR spectra of (a) HUN, (b) $\mathrm{I}_{2 \mathrm{a}} @ \mathrm{HUN}$, (c) $\mathrm{I}_{2 \mathrm{c}} @ \mathrm{HUN}$.
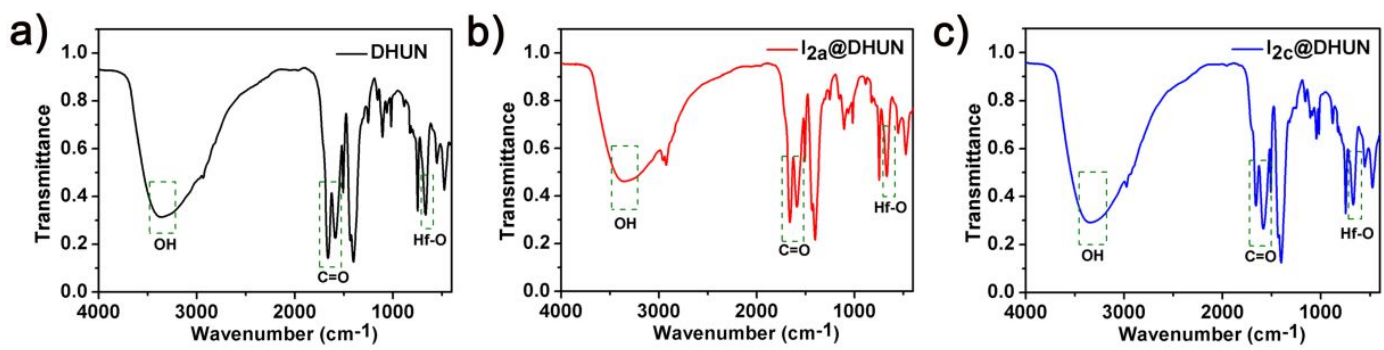

Figure S14. FTIR spectra of (a) DHUN, (b) I $\mathrm{2}_{\mathrm{a}} @ D H U N$, (c) $\mathrm{I}_{2 \mathrm{c}} @ D H U N$. 


\section{Reference}

1. X. Zhu, J. Gu, Y. Wang, B. Li, Y. Li, W. Zhao and J. Shi, Chemical communications, 2014, 50, 8779-8782.

2. G. Lv, J. Liu, Z. Xiong, Z. Zhang and Z. Guan, Journal of Chemical \& Engineering Data, 2016, 61, 3868-3876. 